\title{
A note on the overlap region in turbulent boundary layers
}

\author{
By Jens M. Österlund ${ }^{1}$, Arne V. Johansson ${ }^{1}$, \\ Hassan M. Nagib ${ }^{2}$ \& Michael H. Hites ${ }^{2}$ \\ ${ }^{1}$ Dept. of Mechanics, KTH, SE-100 44 Stockholm, Sweden \\ ${ }^{2}$ Illinois Institute of Technology, Chicago, IL 60616, USA
}

Accepted for publication in Phys. of Fluids

Two independent experimental investigations of the behavior of turbulent boundary layers with increasing Reynolds number were recently completed. The experiments were performed in two facilities, the MTL wind tunnel at KTH and the NDF wind tunnel at IIT. Both experiments utilized oil-film interferometry to obtain an independent measure of the wall-shear stress. A collaborative study by the principals of the two experiments, aimed at understanding the characteristics of the overlap region between the inner and outer parts of the boundary layer, has just been completed. The results are summarized here, utilizing the profiles of the mean velocity, for Reynolds numbers based on the momentum thickness ranging from 2,500 to 27,000. Contrary to the conclusions of some earlier publications, careful analysis of the data reveals no significant Reynolds number dependence for the parameters describing the overlap region using the classical logarithmic relation. However, the data analysis demonstrates that the viscous influence extends within the buffer region to $y^{+} \approx 200$, compared to the previously assumed limit of $y^{+} \approx 50$. Therefore, the lowest $R e_{\theta}$ value where a significant logarithmic overlap region exists is about 6,000 . This probably explains why a Reynolds number dependence had been found from the data analysis of many previous experiments. The parameters of the logarithmic overlap region are found to be constant and are estimated to be: $\kappa=0.38, B=4.1$ and $B_{1}=3.6\left(\delta=\delta_{95}\right)$.

In the classical theory, the overall description of a turbulent boundary layer is dependent on two separate inner and outer length scales. The outer length scale is commonly taken as the thickness of the boundary layer $\delta$, and the inner length scale as the viscous length $l^{*}=\nu / u_{\tau}$, where $u_{\tau}=\sqrt{\tau_{w} / \rho}$ is the friction velocity, $\tau_{w}$ is the skin friction and $\rho$ is the density of the air. Dimensional analysis of the dynamic equations with boundary conditions leads to a scaling of the mean velocity profile in the inner and the outer parts of the boundary 
layer in the form:

$$
\begin{gathered}
\bar{U}^{+}=\frac{\bar{U}}{u_{\tau}}=f\left(y^{+}\right) ; \quad \bar{y}^{+}=\frac{y u_{\tau}}{\nu} \\
\frac{U_{\infty}-\bar{U}}{u_{\tau}}=F(\eta) ; \quad \eta=\frac{y}{\delta}
\end{gathered}
$$

At sufficiently large Reynolds numbers, it is assumed that there is a region of overlap, $\nu / u_{\tau} \ll y \ll \delta$, where the law of the wall (1) and the defect law (2) simultaneously hold. Matching (Millikan 1938) the relations (1) and (2) gives one of the classical results in turbulence theory, i.e., the logarithmic overlap region: in inner variables,

$$
\bar{U}^{+}=\frac{1}{\kappa} \ln \left(y^{+}\right)+B
$$

and in outer variables

$$
\frac{U_{\infty}-\bar{U}}{u_{\tau}}=-\frac{1}{\kappa} \ln (\eta)+B_{1}
$$

By combining Equations (3) and (4) one obtains the logarithmic skin friction law

$$
\frac{U_{\infty}}{u_{\tau}}=\frac{1}{\kappa} \ln \left(\frac{\delta u_{\tau}}{\nu}\right)+B+B_{1}
$$

Recently, due primarily to inconsistencies with trends of experimental data, several researchers have investigated alternatives to the classical theory Barenblatt (1993); George et al. (1997); Zagarola \& Smits (1998).

Based on extensive data from two independent experiments, this investigation targets three main issues related to the overlap region between the inner and outer parts of turbulent boundary layers under zero pressure gradient: the functional form of the overlap, the extent of the overlap and any Reynolds number dependence that may exist in the overlap parameters.

The experiments were carried out in the MTL wind tunnel Johansson (1992) at the department of mechanics, KTH and the NDF wind tunnel at IIT. At KTH, a seven-meter long flat plate was mounted in the test section of the MTL wind tunnel. Measurements of the turbulent boundary layer were performed at five different streamwise stations, $x=1.5,2.5,3.5,4.5$, and 5.5 $\mathrm{m}$ for ten different speeds. At IIT, a $9 \mathrm{~m}$ long and $0.457 \mathrm{~m}$ diameter cylinder was mounted in the test section of the NDF-tunnel Hites (1997); Ornt (1999). Measurements were taken at $\mathrm{x}=1.84,3.65,7.33 \mathrm{~m}$ using five free-stream velocities. In both experiments, the measurement of the velocity profiles was done using hot-wire techniques, the skin friction was measured using oil-film interferometry, and the Reynolds numbers based on the momentum thickness ranged from 2,500 to 27,000 . 


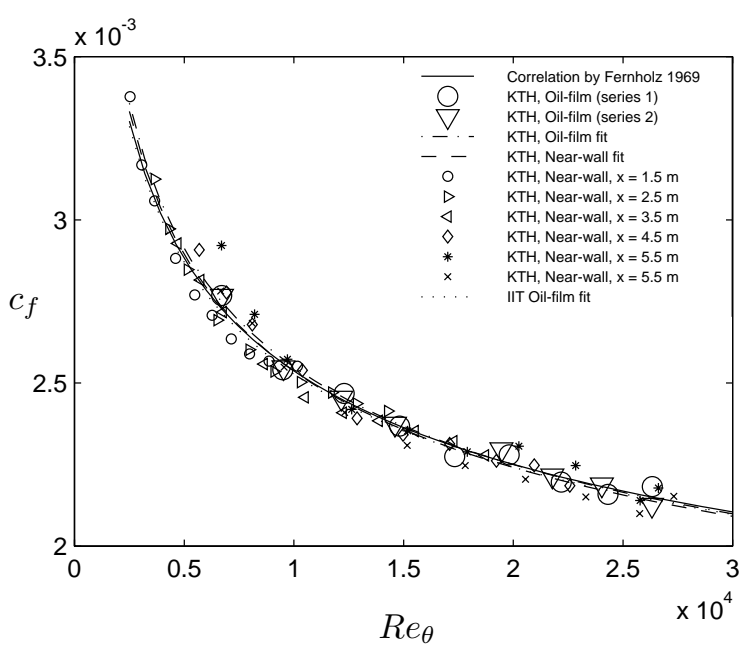

FIGURE 1. Skin-friction coefficient using the oil-film and nearwall methods Österlund (1999), shown with best-fit logarithmic friction laws from Equation (6) and the correlation by Fernholz (1971).

The skin-friction was measured independently of the velocity measurements using oil-film interferometry in a set-up similar to Fernholz et al. (1996), see Fig. 1. The reproducibility of $c_{f}$ obtained with this technique was $\pm 1 \%$. A fit to $c_{f}$ by a variant of the logarithmic skin friction Law (5), namely

$$
c_{f}=2\left[\frac{1}{\kappa} \ln \left(R e_{\theta}\right)+C\right]^{-2},
$$

was made and the friction velocity used in scaling the data was calculated as $u_{\tau}=U_{\infty}\left(c_{f} / 2\right)^{1 / 2}$. The value of the von Kármán constant determined in this way was, $\kappa=0.384$ and additive constant, $C=4.08$. However, it is not possible to determine the additive constants $B$ and $B_{1}$ by this method. In Fig. 1, the results from the oil-film measurements together with the values of the skin friction determined from the mean velocity by the near-wall technique Österlund (1999), are shown together with the calculated best fits using Equation (6). The determined logarithmic skin-friction laws agree very well with each other and also with the correlation developed by Fernholz (1971).

In order to investigate the scaling in the overlap, a normalized slope of the mean velocity profile,

$$
\Xi=\left(y^{+} \frac{d \bar{U}^{+}}{d y^{+}}\right)^{-1}
$$




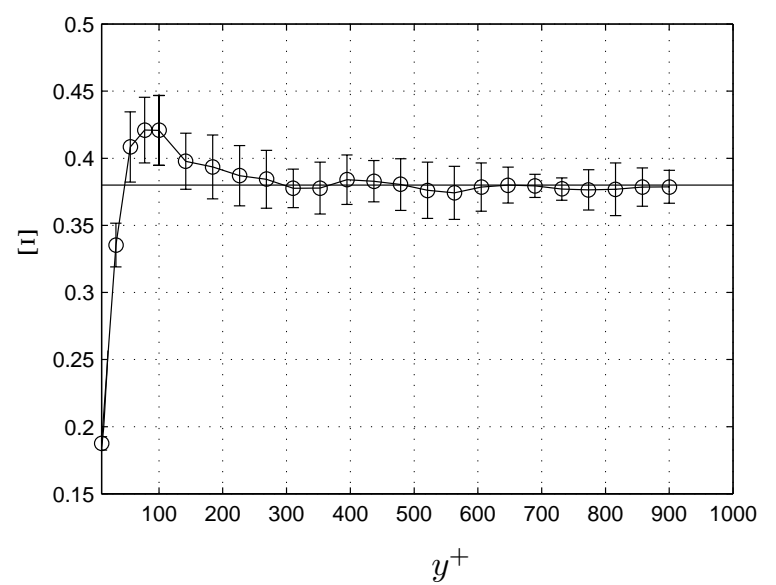

FIGURE 2. Normalized slope of mean profile, $\Xi$, shown in inner scaling; only the part of the profiles in which $\eta<0.15$ was used and the horizontal line corresponds to $\kappa=0.38$

was utilized. In a logarithmic region of the profiles $\Xi$ is constant and equal to $\kappa$. The value of $\Xi$ was calculated by taking an average of the individual profiles at a constant wall distance in inner scaling while omitting the part of the profiles where $\eta>M_{o}$. Similarly, the profiles were again averaged at constant outer-scaled distances from the wall for $y^{+}>M_{i}$. The parameters $M_{i}$ and $M_{o}$ are the inner and outer limits of the overlap region. In Figs. 2 and 3 , the $\Xi$ values averaged over all Reynolds numbers for the KTH data are shown together with error bars representing a $95 \%$ confidence interval. A region where a nearly constant $\Xi$ very accurately represents the data is evident in both figures. This clearly supports the existence of a logarithmic overlap region within the appropriate range of the parameters $M_{i}$ and $M_{o}$. The choice of the appropriate limits was subsequently selected based on the y values where the error bar deviates significantly from the horizontal line in the figures. This was based on an iteration of the limits until a consistent result was obtained. The resulting values for the inner and outer limits are $M_{i} \approx 200$ and $M_{o} \approx 0.15$, respectively. Taking $\kappa$ as the average value within the determined limits gives a $\kappa$ of about 0.38 .

Next, the additive constant B was investigated by looking at the deviation of the mean velocity from the log-function with the aid of the variable $\Psi$, where

$$
\Psi=\bar{U}^{+}-\frac{1}{\kappa} \ln y^{+} .
$$

The variable $\Psi$ is also constant in a region governed by a logarithmic law. The average of the value of $\Psi$ at a constant wall distance is taken for all Reynolds numbers while omitting the part of the profile where $\eta>M_{o}$. In Fig. 4, $\Psi$ 


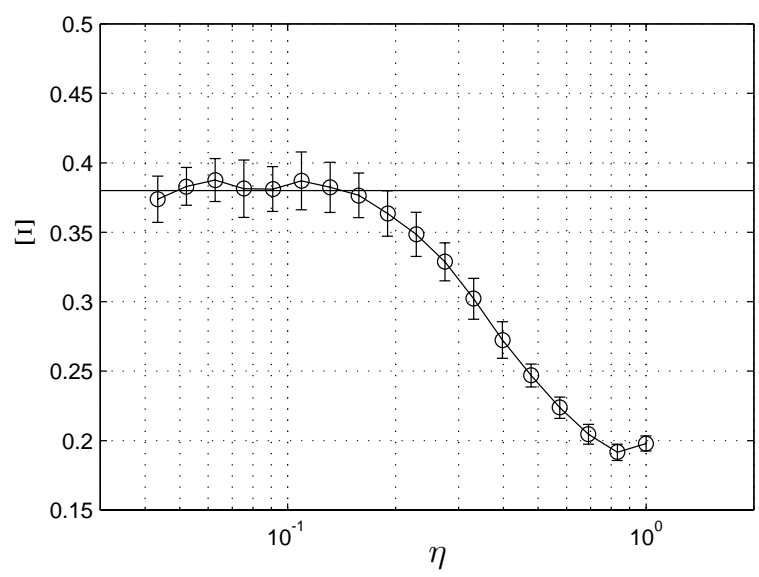

FIGURE 3. Normalized slope of mean profile, $\Xi$, shown in outer scaling; only the part of the profiles in which $y^{+}>200$ was used and the horizontal line corresponds to $\kappa=0.38$

averaged over all Reynolds numbers for the KTH data is shown with error bars corresponding to a $95 \%$ confidence interval. A constant value is found over a wide range in $y^{+}$, again indicating a log-layer. Calculating the average of $\Psi$ within the proposed limits, $M_{i}$ and $M_{o}$, gives $B=4.1$. A slight under-shoot can be seen around $y^{+}=200$ this was found to be caused by a slight Reynolds number variation of $B$ in the lower part of the range. Using only Reynolds numbers above $8-10 \times 10^{3}$ eliminates the small under-shoot.

In addition to using the above described method to determine the loglaw constants, we used the traditional procedure to determine $\kappa$ and $\mathrm{B}$ by performing a least-squares type of fit to the mean velocity profiles. In Fig. 5, $\kappa$ was calculated by fitting a log-law relation for each profile using the following traditional limits of the fit: $M_{i}=50$ and $M_{o}=0.15$. The process was also repeated with the newly established limits of $M_{i}=200$ and $M_{o}=0.15$. The value of $\kappa$ obtained when using the traditional limits varies with Reynolds number and gives about the commonly used value of 0.41 at low Reynolds numbers. Using the new limits, that are more representative of the logarithmic law, again yields a value of $\kappa \approx 0.38$ independent of Reynolds number.

To investigate the existence of a power-law as proposed recently by several authors Barenblatt (1993); George et al. (1997), the following diagnostic function averaged for the KTH data is shown in Fig. 6:

$$
\Gamma=\frac{y^{+}}{\bar{U}^{+}} \frac{d \bar{U}^{+}}{d y^{+}}
$$




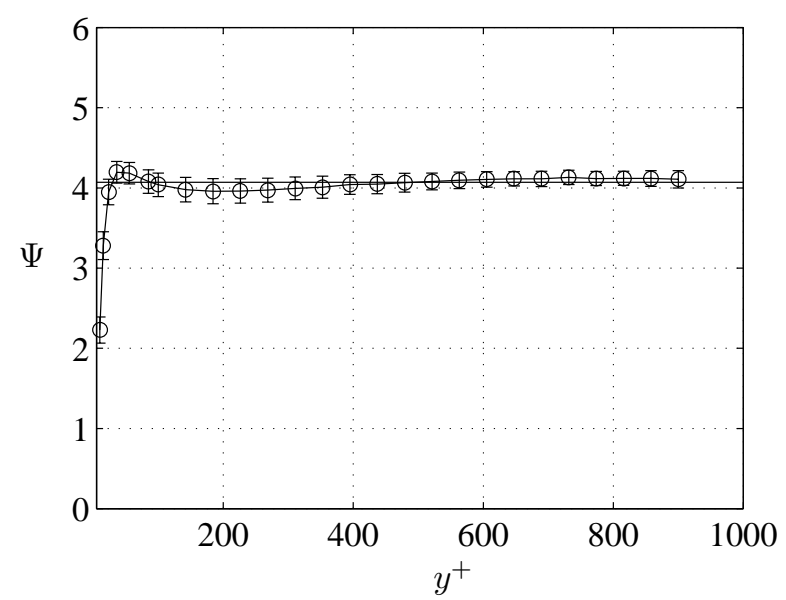

FIGURE 4. Deviation from the logarithmic function using $\kappa=$ 0.38 ; the horizontal line correspond to $B=4.1$, and only the part of the profiles in which $\eta<0.15$ is shown.

The function $\Gamma$ should be a constant in a region governed by a power-law. However, no region of constant $\Gamma$ is depicted in Fig. 6, in particular when compared to Figs. 2 and 3. This clearly indicates that a power-law relation is less representative of the entire region of overlap between $M_{i}$ and $M_{o}$.

Therefore, based on analysis of data from two recent experimental investigations it can be concluded that a logarithmic overlap region, between the inner and outer parts of the mean velocity profiles, exists for $R e_{\theta}>6,000$. Establishing, based on the analysis of the data, an inner limit of the region at about $y^{+}=200$ and an outer limit at $\eta=0.15$ demonstrated the validity of the logarithmic relation with $\kappa=0.38, B=4.1$ and $B_{1}=3.6\left(\delta=\delta_{95}\right)$. The data will be made available in electronic form (http://www.mech.kth.se/ jens/zpg/). 


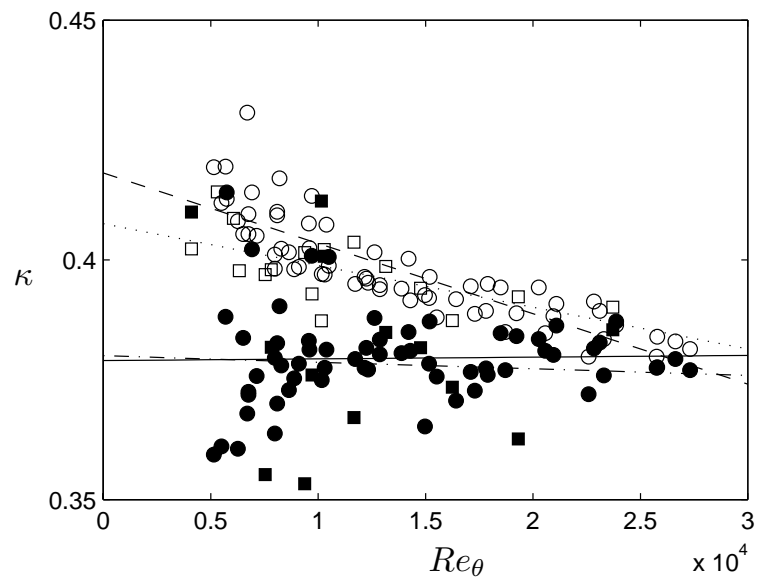

Figure 5. The von Kármán constant determined by a leastsquares fit, with the outer limit fixed at $\eta=0.15$ and the inner limit at $M_{i}$; ○: KTH, $M_{i}=50$. •: $\mathrm{KTH}, M_{i}=200$. Dashed line: KTH, linear fit, $M_{i}=50$. Solid line: KTH, linear fit, $M_{i}=200$. $\square$ : IIT, $M_{i}=50$. $\mathbf{~ : ~ I I T , ~} M_{i}=200$. Dotted line: IIT, linear fit, $M_{i}=50$. Dash dotted line: IIT, linear fit, $M_{i}=200$.

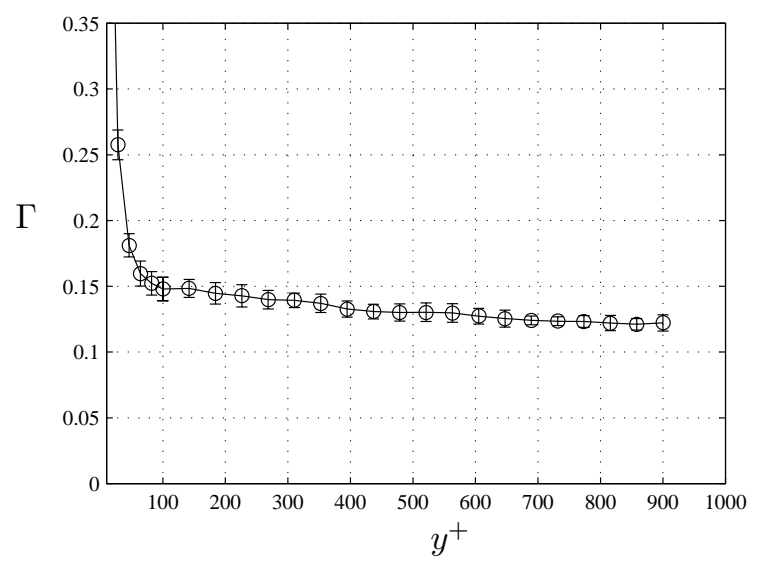

FIGURE 6 . The power-law diagnostic function $\Gamma$; only the part of the profiles in which $\eta<0.15$ is shown. 


\section{References}

Barenblatt, G. I. 1993 Scaling laws for fully developed turbulent shear flows. part 1. basic hypotheses and analysis. J. Fluid Mech. 248, 513-520.

Fernholz, H. H. 1971 Ein halbempirisches Gezetz für die Wandreibung in kompressiblen turbulenten Grenzschichten bei isothermer und adiabater Wand. ZAMM 51, 148-149.

Fernholz, H. H., Janke, G., Schober, M., Wagner, P. M. \& Warnack, D. 1996 New developments and applications of skin-friction measuring techniques. Meas. Sci. Technol. 7, 1396-1409.

George, W. K., Castilio, L. \& Wosnik, M. 1997 Zero-pressure-gradient turbulent boundary layer. Applied Mech. Reviews 50, 689-729.

Hites, M. H. 1997 Scaling of high-reynolds number turbulent boundary layers in the national diagnostic facility. $\mathrm{PhD}$ thesis, Illinois Institute of Technology.

Johansson, A. V. 1992 A low speed wind-tunnel with extreme flow quality - design and tests. In Prog. ICAS congress 1992, pp. 1603-1611. ICAS-92-3.8.1.

Millikan, C. B. 1938 A critical discussion of turbulent flows in channels and circular tubes. In Proceedings of the Fifth International Congress of applied Mechanics.

ORNT, W. 1999 Measurements of wall-shear stress in turbulent channel and boundary layer flows. Master's thesis, Illinois Institute of Technology.

Österlund, J. M. 1999 Experimental studies of zero pressure-gradient turbulent boundary-layer flow. PhD thesis, Department of Mechanics, Royal Institute of Technology, Stockholm.

Zagarola, M. V. \& Smits, A. J. 1998 Mean-flow scaling of turbulent pipe flow. J. Fluid Mech. 373, 33-79. 